\title{
Entropy Generation Minimization for the Optimal Design of the Fluid Distribution System in a Circular MCFC
}

\author{
A. Sciacovelli ${ }^{1} *$, V. Verda ${ }^{1}$ \\ ${ }^{1}$ Department of Energy Engineering, Politecnico di Torino, Turin, Italy \\ E-mail: adriano.sciacovelli@polito.it, vittorio.verda@polito.it
}

\begin{abstract}
In this paper, the prototype of a circular Molten Carbonate Fuel Cell (MCFC) built in the laboratories of Fabbricazioni Nucleari (FN) is analyzed using a tridimensional computational fluid dynamic (CFD) model. This model considers heat, mass and current transfer as well as chemical and electrochemical reactions. The results show that some inhomogeneous distributions in the reactants cause non optimal use of the reactant surfaces. An effective way to improve the distribution in current density consists in tracing tree shaped channels on the surface onto the distribution porous medium. A preliminary study on the effectiveness of such a technical solution is investigated considering a network model of the fluid flow in the porous medium and the channels. Then the optimal shape of distribution channels is investigated considering some geometrical parameters identifying their topology and length as the independent design variables. Minimum entropy generation is considered as the objective function. The results show that significant improvements in the current density can be achieved.
\end{abstract}

Keywords: Molten Carbonate Fuel Cell; entropy generation; optimization.

\section{Introduction}

Among high temperature fuel cells, Molten Carbonate Fuel Cells (MCFC) have reached an advanced state of industrial development. In particular, the integration of this technology with microturbines, which represents a major option for future distributed generation, has been already obtained in various full scale demonstration plants (NELT, 2004). Despite these results, large efforts are still required to improve performances and reliability and to reduce costs.

Numerical modeling is a useful approach to face design problems. Detailed models have been used to understand fuel and oxidant distribution in multi-channels cells (Ma et al, 2003), effects of internal reforming (Park et al, 2002), and ions dynamics (Bittanti et al, 2006). Fluid-dynamic analysis has also been performed in order to study current collectors (Marra, 2008) and simulate electrochemical kinetics (Fontes et al, 1995). Plant energetic and system optimization incorporating MCFC power units has been also tackled, in particular cogeneration (Cigolotti et al, 2008) and electricity-hydrogen production systems (Verda and Nicolin, 2010).

This paper is focused on fuel cell performance improvements through re-design of the fuel distribution system. A circular shaped fuel cell prototype is considered as the application.

The analysis is conducted using a CFD model. Model results are presented for the current design of the distribution system in order to show the inefficiencies and to define possible ways to improve it. The optimization is then conducted by modifying the configuration of the distribution system, with the aim of maximizing the homogeneity of the reactants concentration on the active surfaces.

A fluid network model is used to select the configuration of the fluid distribution, and then an optimization is conducted using the CFD model by varying the geometrical parameters of the selected configuration.

Design methods based on the Second Law of Thermodynamics have been largely applied to the optimization of energy systems. Indeed, reduced entropy generation results in a more efficient design, therefore entropy generation minimization has become popular in the thermo-fluid area. The work by Bejan (Bejan, 1996) is a milestone in this field. He has developed the theoretical aspects as well as the analysis of several applications, mainly treated in analytical form. Numerical analysis of entropy generation has been then successfully applied to different physical phenomena such as two-phase flows (Revellin et al, 2009), MHD flows (Ibanez and Cuevas, 2010), combustion processes (Raghavan et al, 2007) as well as to the design of components such as heat exchangers (San, 2010), fuel cells (Sciacovelli and Verda, 2009), turbomachinery (Iandoli and Sciubba, 2005).

In this paper, entropy generation analysis is applied to a molten carbonate fuel cell prototype manufactured in the laboratories of Fabbricazioni Nucleari (FN) (Amelio et al, 2009). Entropy production is properly formulated in order to take the different transport phenomena occurring in the MCFC into account. The global entropy generation is then adopted as objective function in order to improve gas distribution within the fuel cell. Furthermore, the transport phenomena that mainly affect MCFC performance are highlighted by means of the entropy generation analysis.

\section{System Geometry}

Figure 1 shows a schematic of the FN circular shaped fuel cell. A cross section (A-A) is shown in Figure 2. The cell consists of an external supporting structure in stainless steel, where axial distribution channels are obtained, and the 
fuel cell. 16 distribution channels are used to supply reactants to the cell (4 channels for fuel supply and 4 channels for air supply) and to evacuate the corresponding exhaust flows (4 channels for depleted fuel evacuation and 4 channels for exhaust air evacuation). The fuel cell consists of 9 functional layers plus 2 external supporting layers in stainless steel. Starting from the top, the first functional layer is a porous distribution channel where fuel enters from four radial holes at $90^{\circ}$.

This fluid distribution system allows one to create stacks by simply laying the desired number of cells, one cell upon another.

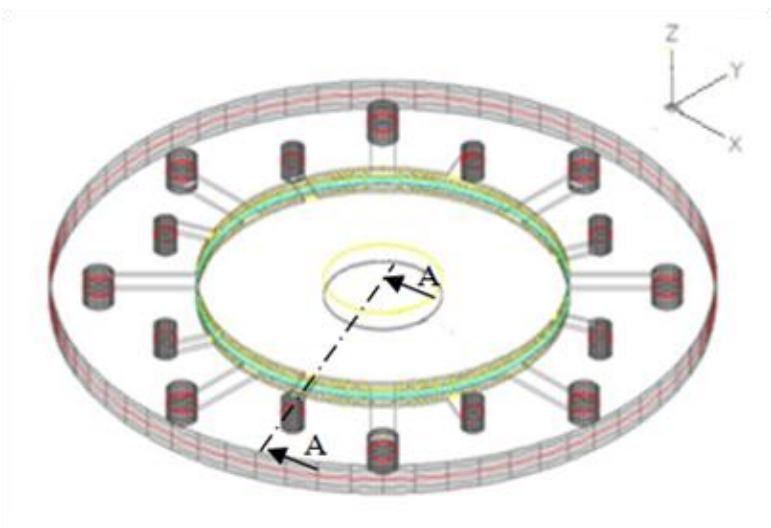

Figure 1. Schematic of a single fuel cell.

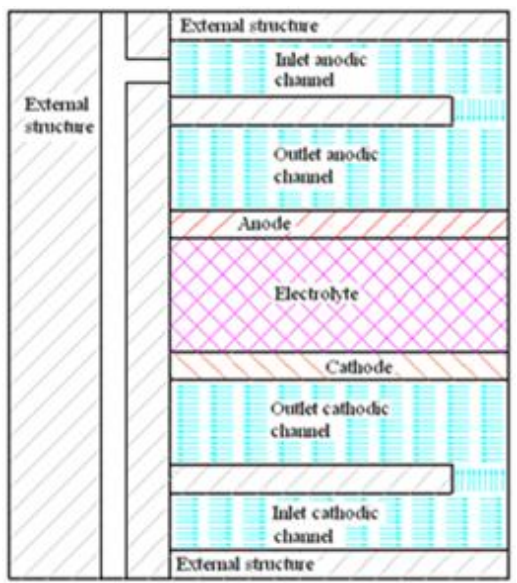

Figure 2. Cross section A-A of the fuel cell.

The fuel in this layer flows from the periphery towards the center. The second layer is a stainless steel plate; some axial holes are obtained in the central part in order to allow the fuel to flow through and reach the lower layer. In the third layer, which is called the outlet anodic channel in Figure 2, fuel flows from the center towards the periphery and permeates the porous anode (fourth layer). As the thickness of the outlet channel is larger than the inlet channel, here the average velocity is smaller. The four outlet holes are at $22.5^{\circ}$ with respect to the inlet holes.

The air distribution is operated in a similar way on the other side of the fuel cell. The air inlet and outlet holes are at $45^{\circ}$ with respect to the inlet and outlet fuel holes.

From physical viewpoint, the only function of inlet channels, both anodic and cathodic, is to convey fuel and air from the peripheral longitudinal ducts toward the center of the fuel cell. Here the gases reverse their direction and flow through the outlet channels providing the reactants to the electrochemical reactions. These reactions take place at the three-phase boundary (TPB): an interface between the porous electrode, where fluid and electrons are transferred, and the electrolyte, where ions are transferred. Gas composition significantly varies along the outlet channels, since the mass source terms are different than zero in the TPB. Accordingly, there are significant heat sources in the TPB.

These features allow one to simplify the complex geometry of Figure 2 in CFD models, when single cells are considered. The simplified geometry represented in Figure 3 and Figure 4 can be analyzed. The central part of the cell, corresponding to the inner edge of the stainless-steel plate separating inlet channel from outlet channel can be neglected. This simplification is possible since the active area of the central part of the fuel cell is relatively small if compared with the remaining portion. With this assumption a further simplification can be adopted: the inlet channels can be substituted by proper boundary conditions on the inlet sections of the anodic and cathodic channels. Since gas composition does not vary along the inlet channel, mass flow rate and species concentrations can be fixed at the inlet sections. Similarly, when a single cell is studied, the representation of the external structure of Fig. 2 can be avoided. Again, proper boundary conditions must be applied. In this case, ambient pressure can be specified on the outlet sections. Finally, from Figure 1 it comes that geometry has a periodic structure, specifically the fuel cell presents a repeating geometrical sector of $90^{\circ}$. So the fuel cell is fully characterized by this portion of the entire geometry.

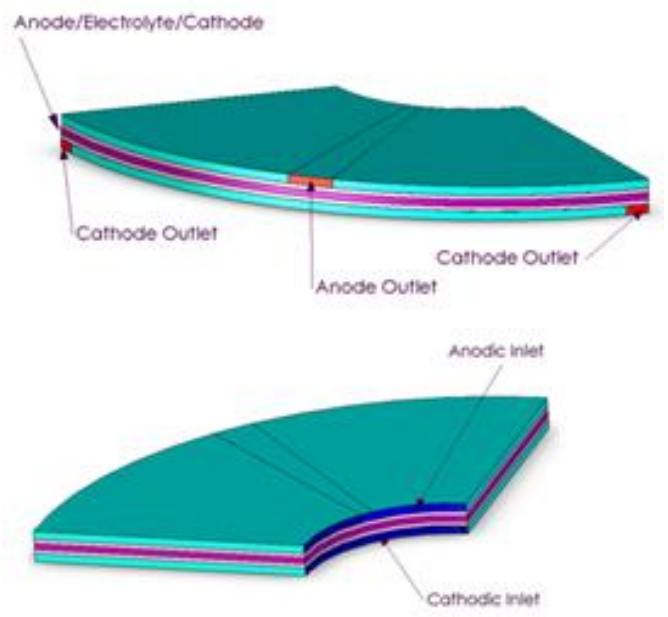

Figure 3. Computational domain.

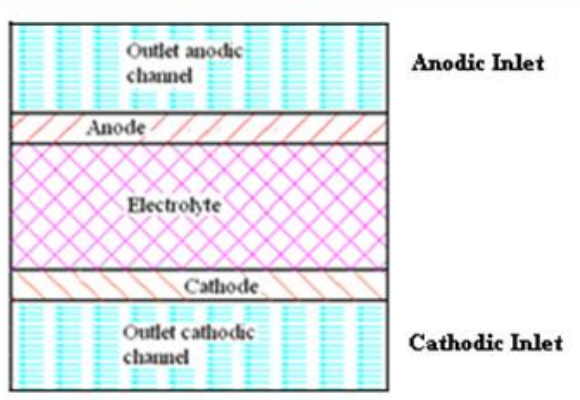

Figure 4. Cross section of the computational domain.

\section{Fuel Cell Model}

The electrolyte of a Molten Carbonate Fuel Cell (MCFC) is constituted of a ceramic matrix filled with sodium and potassium carbonates. At the cathodic side, the porous electrode is permeated by oxidizing gas species, i.e. oxygen and carbon dioxide. Carbonate ions are produced on the 
active surface according with the electrochemical cathode semi-reaction:

$\frac{1}{2} \mathrm{O}_{2}+\mathrm{CO}_{2}+2 e^{-} \rightarrow \mathrm{CO}_{3}^{-}$

Ions migrate through the electrolyte to the anodic side. The anode is fed by hydrogen which reacts with the carbonate ions coming from the cathode, generating water:

$\mathrm{H}_{2}+\mathrm{CO}_{3}^{--} \rightarrow \mathrm{H}_{2} \mathrm{O}+\mathrm{CO}_{2}+2 \mathrm{e}^{-}$

The model here presented consists of a set of partial differential equations taking the different transport phenomena into account: fluid flow, mass transfer, heat transfer, charge transfer, and electrochemical reactions. The model has been developed accordingly to the following assumptions:

1. Steady state conditions.

2. Gas mixtures are considered as ideal gases.

3. Fluid flow is laminar and incompressible.

4. Porous electrodes are homogeneous and isotropic with uniform morphology properties such as porosity, tortuosity and permeability.

Fluid flow and pressure fields are governed by mass and momentum conservation. These two principles can be mathematically expressed by the continuity equation (3) and, under the preceding assumptions, by the Navier-Stokes equation (4):

$\nabla \cdot \rho \vec{V}=0$

$\rho(\vec{V} \cdot \nabla) \vec{V}=-\nabla p+\mu \nabla^{2} \vec{V}$

The ideal gas state equation can be applied to determine the density in the previous equations.

When porous media such as the anode and cathode are considered, it is necessary to consider the presence of the solid matrix. In particular, the momentum equation must be properly modified; in this paper the Brinkman equation (5) is adopted:

$\rho(\vec{V} \cdot \nabla) \vec{V}=-\nabla p+\mu \nabla^{2} \vec{V}-\frac{\mu}{K} \vec{V}$

where $K$ is the permeability of the porous media and $\vec{V}$ must be interpreted as the filtration velocity also known as the Darcy velocity. The term $\rho(\vec{V} \cdot \nabla) \vec{V}$ is maintained since it facilitates to match solutions in a porous media and in an adjacent viscous flow (Nield \& Bejan, 1999).

The chemical species involved in the MCFC electrochemical reactions are: $\mathrm{H}_{2}, \mathrm{CO}, \mathrm{CO}_{2}, \mathrm{H}_{2} \mathrm{O}$ on the anode side and $\mathrm{O}_{2}, \mathrm{CO}_{2}, \mathrm{~N}_{2}$ on the cathode side. The conservation of each species is expressed by a proper balance equation:

$$
\nabla \cdot\left(\rho \omega_{i} \vec{V}\right)=-\nabla \cdot \vec{J}_{i}+S_{i}
$$

in which $\omega_{i}$ is the mass fraction of the $\mathrm{i}$-th species, $\vec{J}_{i}$ is the diffusive flux and must be computed in order to account for bulk and Knudsen diffusion (Krishna \& Wesselingh, 1996).
The flux for the $\mathrm{i}^{\text {th }}$ species is expressed through the following equation:

$\vec{J}_{i}=$
$-\rho D_{i, e f f} \nabla \omega_{i}+\frac{\rho \omega_{i}}{M}-M \sum_{j} D_{i, e f f} \nabla \omega_{j}-\nabla M \sum_{j} D_{j, e f f} \nabla \omega_{j}$

The first term on the right-hand side represents the Fick diffusion due to concentration gradients. The last three terms are the corrections to enforce the Stefan-Maxwell equations for multi-component diffusion term (Hwang, Chen \& Lai, 2005). The coefficient $D_{i, e f f}$ is the effective binary diffusivity to account for reduced mass flux due to solid structure of the porous medium. The generation/destruction of species due to electrochemical reaction is considered through proper boundary conditions instead of volumetric source term, therefore $S_{i}$ is zero in Eq. (6). This boundary condition is expressed as a flux through Faraday law (Koh, Seo, Yoo \& Lim, 2002).

Heat transfer is solved by means of the energy equation, where the viscous effects have been neglected:

$\nabla \cdot(\vec{V}(\rho E+p))=\nabla \cdot\left(k_{e f f} \nabla T-\sum_{\alpha} h_{\alpha} \vec{J}_{\alpha}\right)+S_{h}$

where $k_{\text {eff }}$ is an average thermal conductivity of the coexisting solid and gas phases. In the porous matrix the local thermal equilibrium hypothesis is assumed, so that a single temperature is used to describe the gas phase and the solid phase. In the electrodes the volumetric heat source term is due to Joule heating effect and is formulated as

$S_{h}=\sigma \nabla \phi \cdot \nabla \phi$

in which $\phi$ is the electric potential and $\sigma$ is the electrical conductivity. Reversible heat associated with chemical reaction and activation loss associated with the electrochemical reactions are accounted through proper boundary conditions on the surfaces between the electrolyte and electrodes. In the electrolyte, only the source term (9) due to Ohmic heating survives.

To solve the electrical problem current density the electric potential must be found. Ohm's law can be used to relate the current density $\vec{\imath}$ to the electric potential:

$\vec{\imath}=-\sigma \nabla \phi$

The potential field throughout the conductive regions is calculated based on the conservation of charge:

$\nabla \cdot \vec{\imath}= \begin{cases}\mathrm{j} & \text { in the TPB } \\ 0 & \text { elsewhere }\end{cases}$

where $j$ is the current generation rate, produced at the TPB. This is assumed as coincident with the surface between the electrolyte and the electrodes. The current source is expressed by the Butler-Volmer equation:

$i=i_{0}\left\{e^{\left[\frac{\beta z F \eta_{a c t}}{R T}\right]}-e^{\left[-\frac{(1-\beta) z F \eta_{a c t}}{R T}\right]}\right\}$

where $\eta_{a c t}$ is the electrode overpotential which is related with the reversible potential (Nernst's potential) and the ohmic losses through the following equation: 
$\eta_{\text {act }}=V_{\text {rev }}-\left|\eta_{\text {electrode }}-\eta_{\text {electrolyte }}\right|$

Numerical independence analysis has been performed in (Verda and Sciacovelli, 2011).

\section{Model Results}

The momentum equation, transport equations and boundary conditions are solved using the finite volume method in order to obtain velocity field, temperature and mass fractions in the fuel cell. The local current density is then computed so that the source term and boundary conditions for species and energy equation are determined. This procedure is repeated until convergence is reached.

Convective terms are computed by second order upwind scheme while the diffusive terms are computed by the central difference scheme. The numerical solution procedure adopts the semi-implicit SIMPLE algorithm. The detailed numerical procedure is available in (Patankar, 1980).

Simulations have been performed considering mass flow rates of $3 \cdot 10^{-6} \mathrm{~kg} / \mathrm{s}$ at the anode (the mass fraction of constituents are $0.3 \mathrm{H}_{2}, 0.4 \mathrm{CO}_{2}, 0.3 \mathrm{H}_{2} \mathrm{O}$ ) and $1 \cdot 10^{-5} \mathrm{~kg} / \mathrm{s}$ at the cathode (the mass fraction of constituents are $0.23 \mathrm{O}_{2}$, $0.23 \mathrm{CO}_{2}, 0.54 \mathrm{~N}_{2}$ ) and an inlet temperature of $823 \mathrm{~K}$.

The velocity field in the anodic outlet channel is represented in Figure 5. Velocity is purely radial in the proximity of the inner radius of the computational domain. As fluid particles move toward the periphery of the cell they assume a circumferential velocity component due to the presence of the outlet section. This velocity component is particularly significant for the gas particles moving close to the outer wall of the fuel cell, where the flow field assumes a pure tangential configuration. Moreover, the fuel does not feed the cell uniformly since the fluid velocity magnitude is not homogenously distributed, as shown in the figure. A similar velocity field occurs in the cathodic compartment.

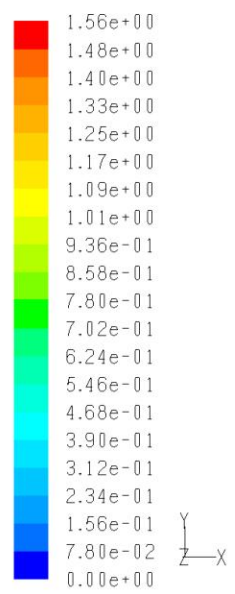

Figure 5. Pathlines in the anodic outlet channel $(\mathrm{m} / \mathrm{s})$ (figure is in color in the on-line version of the paper).

Both in the anodic and cathodic outlet channels there are areas where velocity is very small. This affects the chemical species distribution: hydrogen, oxygen and carbon dioxide mass fractions are small in the areas between the outlet sections.

Hydrogen mass fraction is shown in Figure 6. The largest concentration occurs in the central area, in correspondence of the fuel inlet section. Concentration decreases towards the outlet section because of the fuel consumption associated with the electrochemical reaction. However the decrease of $\mathrm{H}_{2}$ mass fraction is large where the gas velocity is lower, since these regions are reached by a smaller amount of fuel.
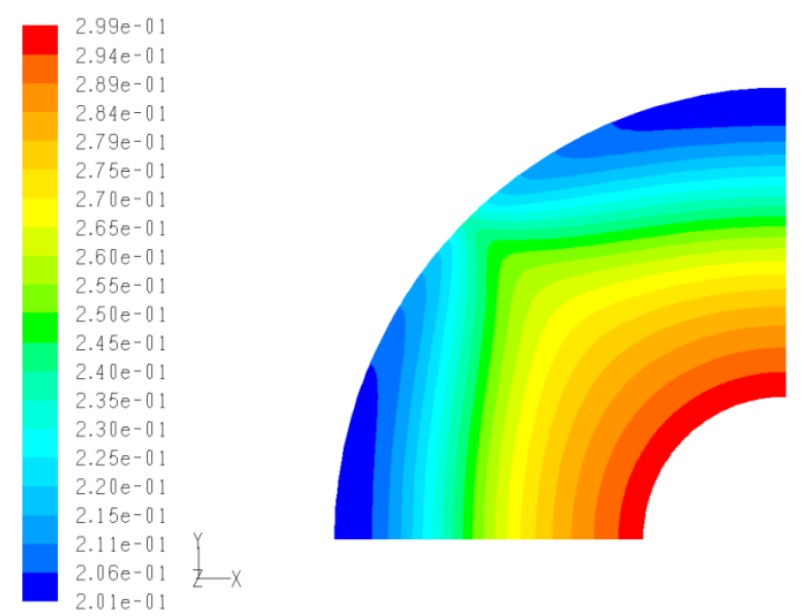

Figure 6. Hydrogen mass fraction (figure is in color in the on-line version of the paper).

Accordingly to the electrochemical reaction (2) water and $\mathrm{CO}_{2}$ are produced at the anodic side of the cell. The corresponding mass fractions increase from the center toward the periphery of the fuel cell. Figure 7 shows the water mass fraction.
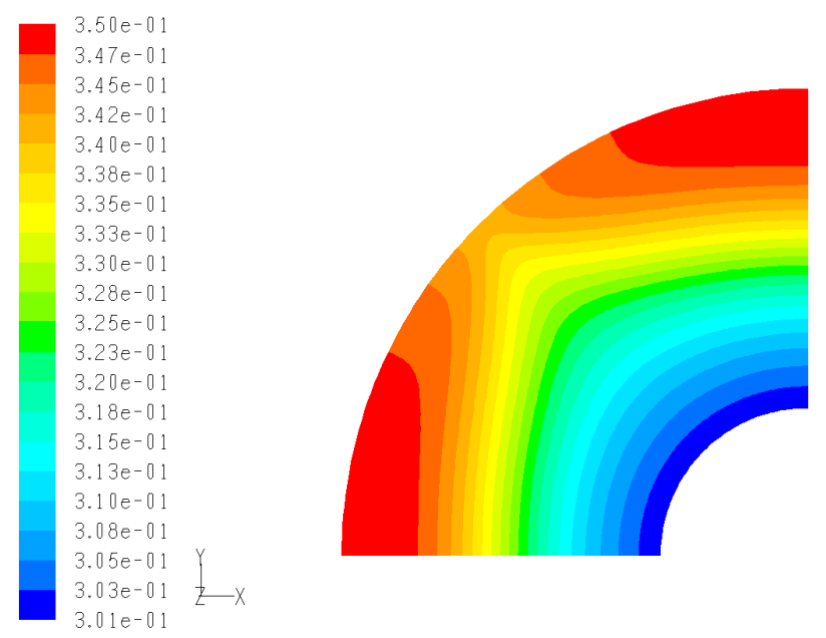

Figure 7. Water mass fraction in the anode (figure is in color in the on-line version of the paper).

The non-uniformities of the velocity field also affect $\mathrm{H}_{2} \mathrm{O}$ and $\mathrm{CO}_{2}$ concentration distribution: where the velocity is high, these species are quickly conveyed toward the outlet; consequently, mass fractions become small. In the stagnation areas there are higher concentrations of these species.

Oxygen mass fraction decreases at the cathodic side of the fuel cell because carbonate ions are produced according with reaction (1). The fuel cell is fed by a higher oxidant mass flow rate compared to fuel mass flow rate; for this reason the decrease of $\mathrm{O}_{2}$ concentration is limited. Oxygen mass fraction is shown in Figure 8.

The current density distribution on the surface between anode and electrolyte is shown in Figure 9. This figure shows that the utilization of the active area rapidly decreases from the center to the periphery because of the decreasing concentration of hydrogen and oxygen. 

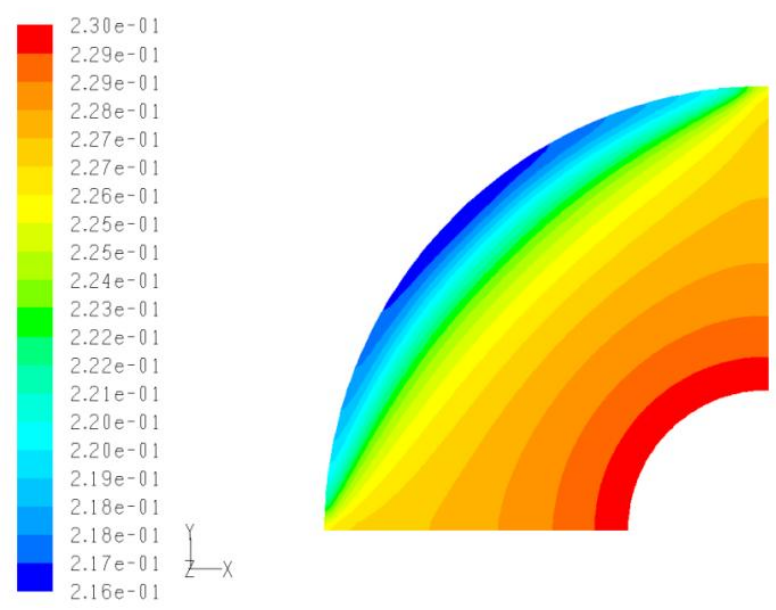

Figure 8. Oxygen mass fraction (figure is in color in the online version of the paper).
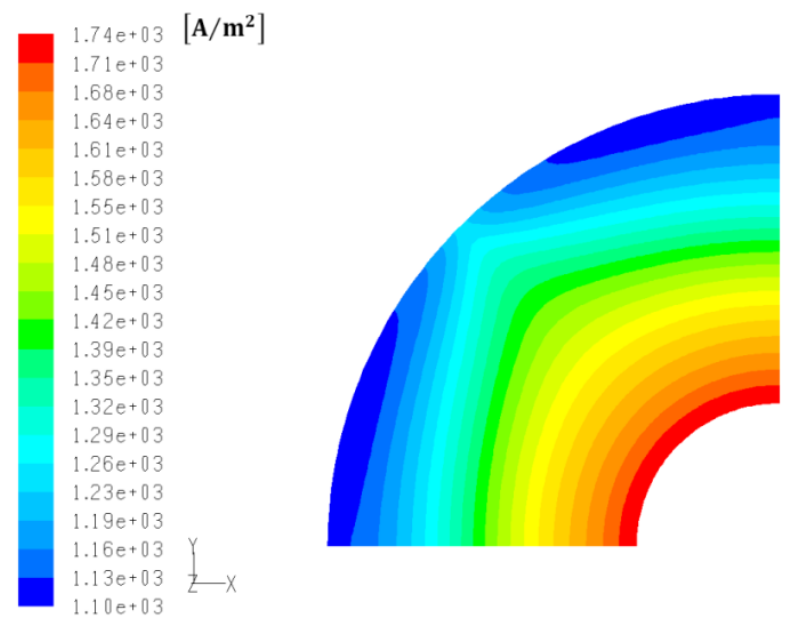

Figure 9. Current density distribution (figure is in color in the on-line version of the paper).

Results of the presented model have been validated in (Massano, Sciacovelli \& Verda, 2009) using some experimental results available from FN corresponding with a stack of 15 cells (Amelio et al, 2009).

\section{Network analysis for design improvement}

To improve the utilization of the active area, two main changes in the distribution system can be operated: 1) the number of outlet channels can be increased in order to make the outlet fluid velocity more homogeneous; 2) radial distribution channels can be traced in order to distribute fresh reactants to a wider area. The first design change involves changes at stack level, and this option is not considered in the present work. Instead, three shaped channels between the metallic structure and the outer part of the anodic and cathodic outlet channels are considered. A complete CFD analysis and optimization of the fuel cell and the fluid distribution structures involve large computational load. For this reason a preliminary design improvement is performed using a fluid dynamic network model. This approach is used to select the fluid distribution configuration (one or two bifurcation levels, as discussed below) that is then optimized using the CFD model.

In the network approach, porous media are discretized in nodes. Adjacent nodes are then connected through onedimensional branches. Similarly, radial channels are represented as one-dimensional branches connecting nodes (Calì \& Borchiellini, 2002). Although this scheme can be used to solve all the phenomena presented in the previous section, here only the fluid dynamics is considered.

Momentum equation for a steady state incompressible flow in a general branch is

$J \cdot l_{a}+J \frac{p_{2}-p_{1}}{\rho}=0$

where $J$ is the mass flow rate, $l_{a}$ the unit mass friction loss and $p$ the static pressure in the initial and final nodes. Kinetic and potential energy differences are considered as negligible. In channels, friction losses are expressed as the summation of distributed and local losses. In both cases the loss can be expressed as the function of the square of the average velocity, or of the square of the mass flow rate, namely:

$l_{a d}+l_{a c}=R_{h} \cdot J^{2}$

where $R_{h}$ is a resistance coefficient. Pressure drop can be obtained from Eqn (15) as

$\Delta p=p_{2}-p_{1}=\rho \cdot R_{h} \cdot J^{2}=s_{12} \cdot R_{p} \cdot J^{2}$

The coefficient $s_{12}$ can have the values +1 and -1 and it takes the true (not arbitrary) verse of the flow into account. Eqn (16) can be rewritten as:

$J=\frac{p_{1}-p_{2}}{R_{p}^{0.5} \cdot|\Delta p|^{0.5}}=Y_{12} \cdot\left(p_{1}-p_{2}\right)$

being $Y_{12}$ the flow conductance of the branch. In the case of the porous medium, the flow conductance is constant and is obtained from the Darcy equation.

To solve the complete network, the momentum equation must be written for all the branches. In addition, the continuity equation must be written for all the nodes. The continuity equation for a node is written as

$-\sum_{i=1}^{n_{\text {in }}} J_{i}+\sum_{i=1}^{n_{\text {out }}} J_{i}+J_{\text {ex }}=0$

where $n_{\text {in }}$ is the number of flows entering the node from branches, $\mathrm{n}_{\text {out }}$ is the number of flows exiting the node and entering branches $\left(n_{\text {in }}+n_{\text {out }}\right.$ is equal to the number of branches having the node as initial or final end) and $\mathrm{J}_{\mathrm{ex}}$ is the mass flow rate exiting the node to the ambient. This term is negative if the flow enters the node from the ambient. The conventional verse of the branches is used to set the equation.

The continuity equation can be written for the whole network in a matrix form. The incidence matrix, $\mathbf{A}$, (see for example (Chandrashekar \& Wong, (1982)) can be adopted to express the network topology. This is characterised by as many rows as the number of nodes $(n)$ and as many columns as the number of branches $(n)$. The element $\mathrm{A}_{\mathrm{ij}}$ is 1 if the branch $j$ exits the node $i,-1$ if the branch $j$ enters the node $i$ and 0 if the node $i$ and the branch $j$ are not related. The continuity equation is written as

$$
\boldsymbol{A} \cdot \boldsymbol{J}+\boldsymbol{J}_{\text {ext }}=0
$$

where $\mathbf{J}$ is the vector ( $m$ rows) of the mass flow rates in the branches and $\mathbf{J}_{\mathrm{ex}}$ the vector ( $n$ rows) of the mass flow rate exiting the nodes to the ambient. Equation (19) can be rearranged by substituting the expression of mass flow rate 
in the branches obtained from the momentum equation. To do so, a matrix expression must be derived. The pressure difference in all the branches can be expressed as

$\Delta \boldsymbol{P}=\boldsymbol{A}^{T} \boldsymbol{P}$

where $\mathbf{P}$ is the vector ( $n$ rows) of the pressure in the nodes. A diagonal matrix $\mathbf{Y}(m \times m)$ is introduced, whose term $Y_{j j}$ is the flow conductance of the $j^{\text {th }}$ branch. The mass flow rate in the branches can be expressed as

$J=Y \cdot \Delta P=Y \cdot A^{T} \cdot P$

and then, substituting in (19)

$$
\left[A \cdot Y \cdot A^{T}\right] \cdot P+J_{e x t}=0
$$

To solve the set of non-linear equations (22) boundary conditions must be set. In this work, the inlet mass flow rate is fixed while a constant pressure is imposed on the active surface.

Two architectures are considered, the first one characterized with a single bifurcation level and the second one with two bifurcation levels (Bejan \& Lorente, 2004). These architectures are shown in Figure 10. The design parameters of three shaped channels, the angles and lengths of ducts, are progressively modified in order to maximise the angular momentum associated with fluid reaching the active surface. The reason for such objective function is that we are interested in increasing as much as possible the use of external area. The analysis is conducted considering the total surface covered by the ducts as constant. In addition, only symmetrical configurations are considered.
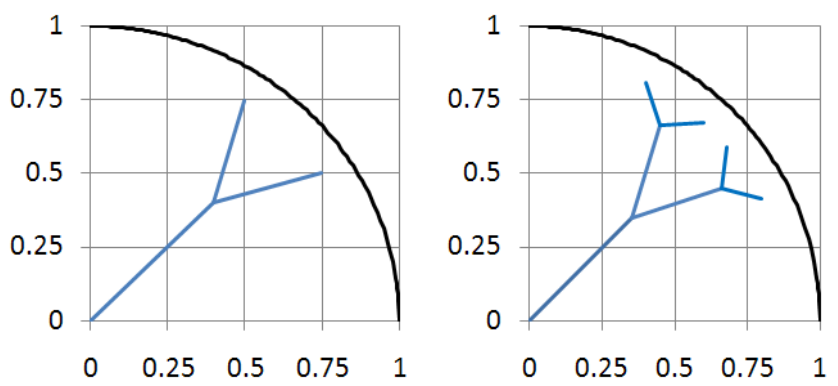

Figure 10. Selected architectures of the three shaped channels.

The results corresponding with the analysis of 100 configurations is presented in Figure 11.

These configurations corresponds to 10 values of the ratio between channel length and 10 values of the bifurcation angle. Blue bars refer to single level bifurcations, while red bars to double level bifurcations. The latter allows better flow distribution, since the angular momentum is larger. On average, the difference between the two architectures is about $11.5 \%$, while the optimal configurations differs by $5.8 \%$ only.

Thanks to this result, it is possible to limit the application of the CFD model and the geometry optimization to the single bifurcation architecture only. The advantage is that the geometrical description of the two bifurcation level is much more complicated, as it involves four design parameters instead of two. These parameters must be varied in the optimization process.

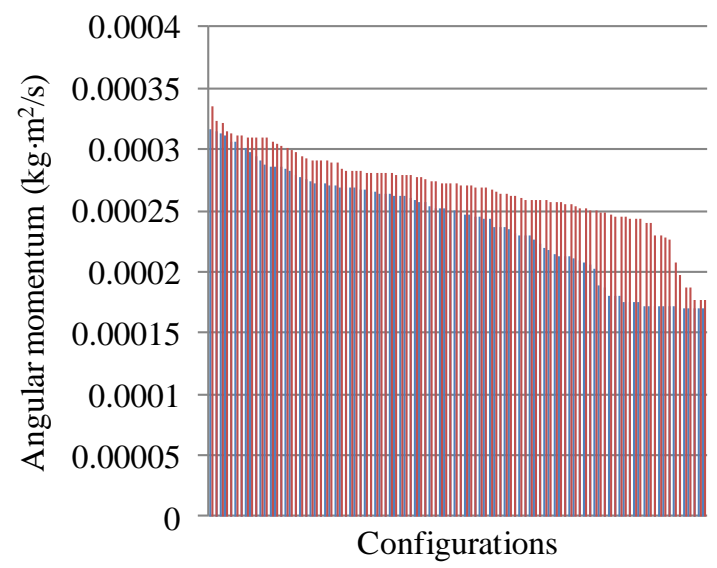

Figure 11. Angular momentum of fluid using three shaped distributed channels.

\section{Geometry Optimization}

The problem of optimal fluid distribution inside a given physical system has been tackled by a large number of researchers; typical examples in engineering are heat exchangers, pipe networks and cooling devices (Zimparov, da Silva \& Bejan, 2006; Copiello \& Fabbri, 2009). Similar studies have been also carried out for different typologies of fuel cells. In this case it is necessary to exploit a given amount of reactants in the most effective way, i.e. to obtain the maximum electric power (Xing, Lum, Poh \& Wu, 2009). The entropy generation rate is one of the possible indicators for choosing the optimal design of an energy system. This quantity can not only be used to express the objective function in the optimization, but also to select the design parameters through analysis of the distribution of the entropy generation terms (Sciacovelli \& Verda, 2009).

As discussed in the previous section, a Y-shaped inlet channel in the anodic compartment is considered. Similar conclusions can be drawn for the cathodic compartment.

The new geometry of the computational domain is shown in Figure 12.

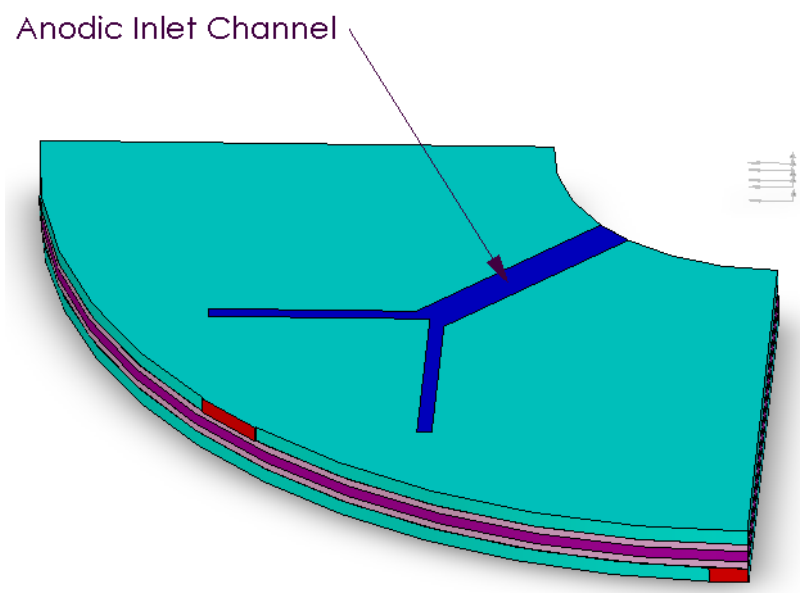

Figure 12. Modified MCFC geometry.

The effect of the Y-shaped channel on the fuel cell performance can be maximized by modifying the dimensions and orientation of the branches. Two antagonistic factors influence the optimal design of gas distribution inside the fuel cell: the maximum electrical power and the maximum efficiency.

In a fuel cell the departure from ideal conditions is due to the so-called polarization losses. These effects are strictly 
related to the irreversible transport phenomena occurring in the system. Thus, the rate of entropy generation is a proper physical quantity that can be utilized to identify what are the major causes of irreversibility and efficiency decrease.

Entropy generation can be expressed in terms of fluxes times the gradients of the corresponding physical quantities. The equation is obtained from the entropy balance written for an infinitesimal volume, that is:

$\rho \frac{D s}{D t}=-\nabla \cdot \vec{\sigma}+g_{p}$

where $s$ is the entropy per unit mass, $\vec{\sigma}$ is the entropy-flux vector and $g_{p}$ is the rate of entropy generation per unit volume. To obtain $\vec{\sigma}$ and $g_{p}$ we can assume local thermodynamic equilibrium, it follows that $s$ is a well defined function of the various properties necessary to define the macroscopic state of the system. This can be expressed considering that the total differential of $s$ is given by the Gibbs relation:

$d u=T d s-p d\left(\frac{1}{\rho}\right)+\sum_{i} \mu_{i} d \omega_{i}$

Differentials can be expressed using energy equation, the conservation of species and the fluid flow equation. After some rearrangement it is possible to show that:

$\vec{\sigma}=\frac{1}{T}\left(\vec{J}_{q}+\sum_{i} \mu_{i} \vec{J}_{i}\right)$

The local rate of entropy generation can be split in four main contributions, each strictly correlates to a specific physical phenomenon:

$g_{p}=g_{\mu}+g_{h}+g_{m}+g_{c}$

The first term at the right-hand side of Eqn. (26) is connected to the gradients of the velocity field, giving rise to viscous flow, the second arises from heat conduction, the third from mass transfer, and the fourth term is due to coupling between heat and mass transfer (convective term). The structure of the expression is that of a bilinear form: it is the summation of a flow quantity times the gradient of an intensive state variable driving that flow:

$g_{p}=\frac{1}{T} \Delta: \tau+\frac{1}{T^{2}}\left(-J_{q} \cdot \nabla T\right)+\frac{1}{T}\left(\sum_{i}-\vec{J}_{l} \cdot \nabla \mu_{i}\right)+$ $\frac{1}{T}\left(\sum_{i}-s_{i} \overrightarrow{J_{l}} \cdot \nabla T\right)$

in which $\Delta$ and $\tau$ are the strain and stress tensor. For a Newtonian fluid they can be expressed in terms of viscosity and fluid velocity gradient. In the case of a fuel cell, an additional term must be considered in Eqn (26). This term is associated with the entropy generation due to ohmic losses:

$g_{o h m}=\frac{1}{T} \sigma \nabla \phi \cdot \nabla \phi$

The previous term is present only in conductive regions, that is electrodes and electrolyte.

Since we are interested in the optimal configuration of the anodic inlet channel, it is necessary to properly parameterize the MCFC geometry in order to define the design variables free to vary. Figure 12 shows that four design parameters can be used to characterize the geometry configuration. Specifically, the lengths $R_{1}$ and $R_{2}$ of two branches and the angles $\theta_{1}$ and $\theta_{2}$ are free to vary. The remaining length $R_{3}$ is chosen in order to keep the total area of the Y-shaped channel constant. If symmetric geometries are consider, the number of free parameters is reduced to two.

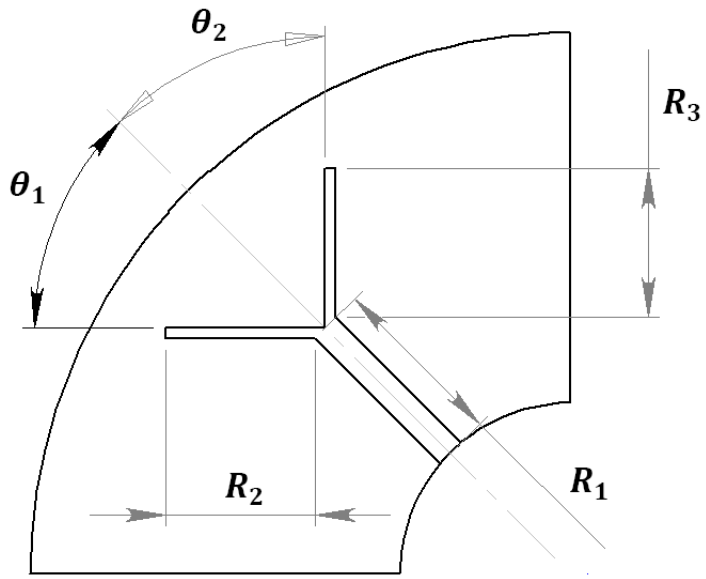

Figure 12. Parameterized MCFC geometry.

The fuel cell optimization is performed by considering two objective functions. The first objective function $F_{1}$ is the global entropy generation rate $G_{p}$, namely

$F_{1}=\int\left(g_{\mu}+g_{h}+g_{m}+g_{c}+g_{o h m}\right) d V$

The second objective function $F_{2}$ is the electric power produced by the fuel cell, that is

$F_{2}=\dot{W}_{e l}$

Clearly it is desirable to maximize the electrical power, i.e. $F_{2}$, and at the same time reduce as much as possible the sources of irreversibilities, that is minimize $F_{1}$. For this reason the optimization has been performed using the Nondominated Sorting Genetic Algorithm version II (NSGA-II) (Kalyanmoy, 2002). This is an evolutionary algorithm suitable for simultaneously optimization of two or more conflicting objectives. NSGA-II uses some mechanisms inspired by biological evolution: reproduction, mutation, recombination, and selection. Candidate solutions to the optimization problem play the role of individuals in a population, and the objective function determines the environment within which the solutions survive. Evolution of the population then takes place after the repeated application of the above operators.

\section{Optimization Results}

Various geometries have been analyzed during optimization. Figure 13 shows the scatter plot of global entropy generation versus power density, the Pareto front of optimal designs are indicated with red squares.

The figure confirms that the two objective functions are conflicting. According to the Pareto curve it is possible to achieve a higher power density to the detriment of a larger value of entropy generation.

The scattered distribution in the figure is due to the high computational costs in the optimization. The Pareto front could be determined with better precision (increased number of points) but this would require a much larger number of 
simulations, without adding a real value to the results. In fact the deviation between minimum and maximum power densities of the points on the Pareto front is about $8 \mathrm{~W} / \mathrm{m}^{2}$, i.e. about $0.6 \%$.

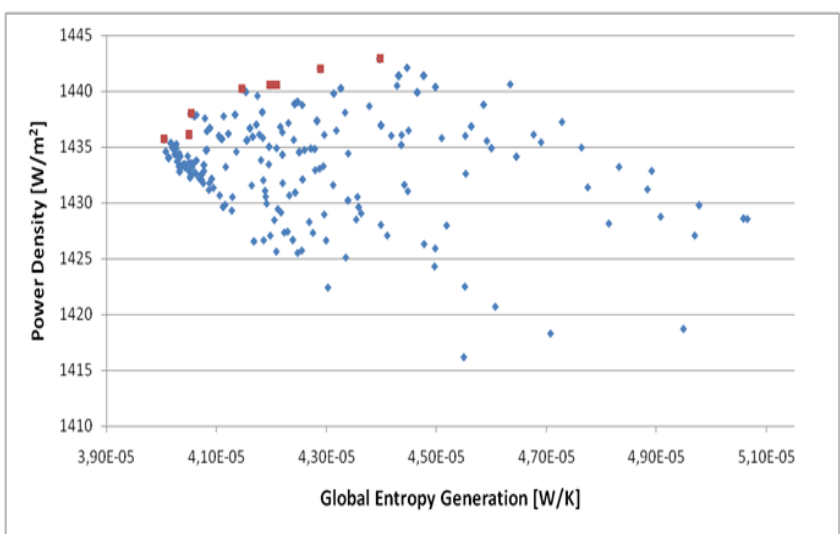

Figure 13. Parameterized MCFC geometry.

The streaks on the figure are due to the evolution in the search for the optimal points. This is conducted by considering discrete values of the independent design parameters. Some auto-correlations may occur due to this discretization. This would not occur in the case of a search in a continuum design space (which is not practically possible to explore because of the too large computational cost).

A bubble chart can be used to understand the influence of each design parameter on the objective functions. This representation is used as the data have four dimensions that need to be shown on the same chart. In Figure 13 the design variables $R_{1}$ and $R_{2}$ are reported along abscissa and ordinate, the color maps shows the power density of the fuel cell, finally the diameter of the bubble indicates the global entropy generation.

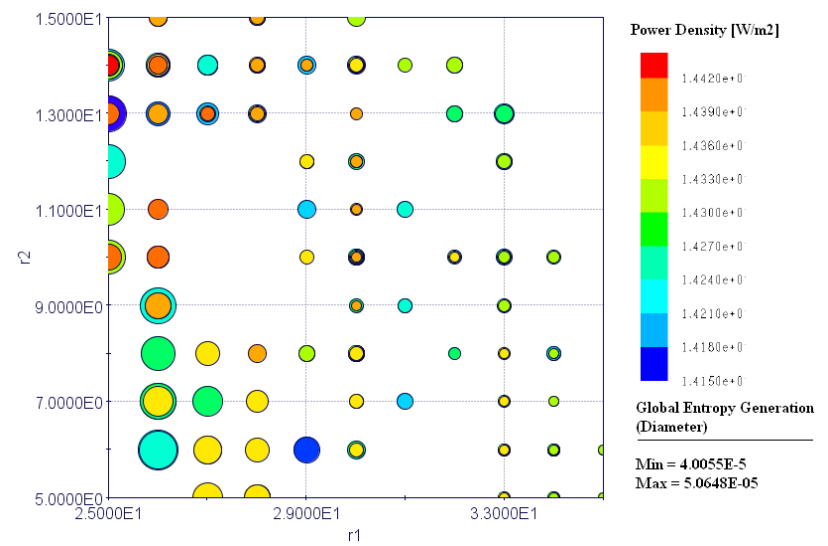

Figure 14. Bubble chart of $R_{1}$ and $R_{2}$ (figure is in color in the on-line version of the paper).

Higher power density is achieved by geometrical configurations with small values of $R_{l}$ and large $R_{2}$. This means that an inlet channel with a short central branch and long secondary branch promotes a higher electrical power. When the opposite situation occurs, that is large $R_{l}$ and small $R_{2}$, the entropy generation is minimized.

This is similar to the result obtained with fluid flow analysis shown in Figure 10. Differences in the optimal lengths are also due to the different position of the inlet section: in the network model the central area is considered, while this is not considered in the three dimensional model.
It is also necessary to understand how the other two design parameters, i.e. the angles $\theta_{1}$ and $\theta_{2}$, affect the objective functions. This is shown in Figure 15.

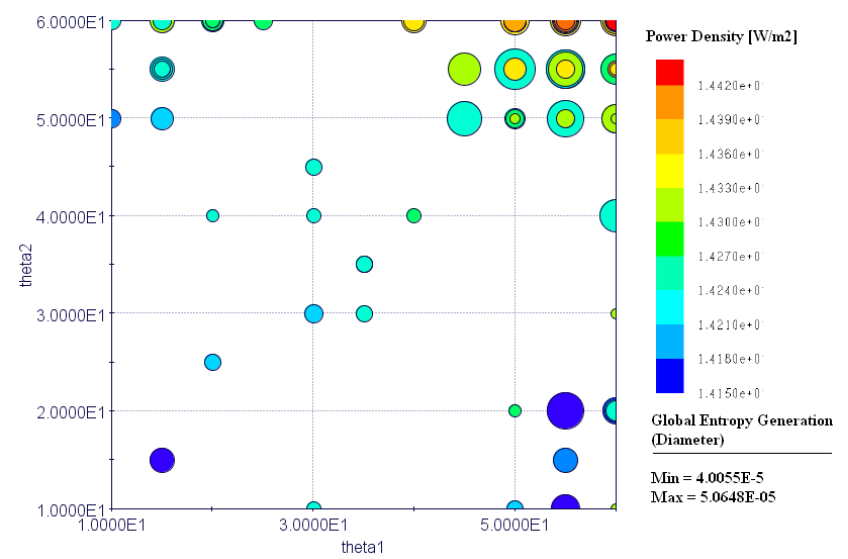

Figure 15. Bubble chart of $\theta_{1}$ and $\theta_{2}$ (figure is in color in the on-line version of the paper).

Large values of the angles $\theta_{1}$ and $\theta_{2}$ allow one to obtain large power densities. Moreover from Figure 15 it is obvious that the two angles should be both large, this also means that a symmetrical anodic inlet channel is preferable when high electric power is required. Conversely, if the Y-shaped channel assumes a close configuration, i.e. small $\theta_{1}$ and $\theta_{2}$, the entropy generation is reduced. The Pareto designs are the best compromise with respect to the two objective functions adopted here. One of these designs is illustrated in Figures 16 and 17 , specifically the case characterized by $R_{l}=14$ $\mathrm{mm}, R_{2}=27 \mathrm{~mm}, \theta_{1}=60^{\circ}$ and $\theta_{2}=60^{\circ}$. Accordingly to the two previous bubble charts, this configuration provides with high power density due to the ample angles. However, the relatively long central branch assures a relatively low global entropy generation.

The current density distribution corresponding with this design is illustrated in Figure 16; the inlet channel outline is highlighted in blue. The presence of the two secondary branches allows one to obtain larger current density, especially for large radius. Moreover in the area spanned by the Y-channel the current density gradients are reduced if compared to the original configuration. This means that in the optimized design more homogeneous operating conditions take place. In the region far from the channel, the current density assumes lower values since this region is fed by partially depleted fuel, that is with a lower hydrogen concentration.
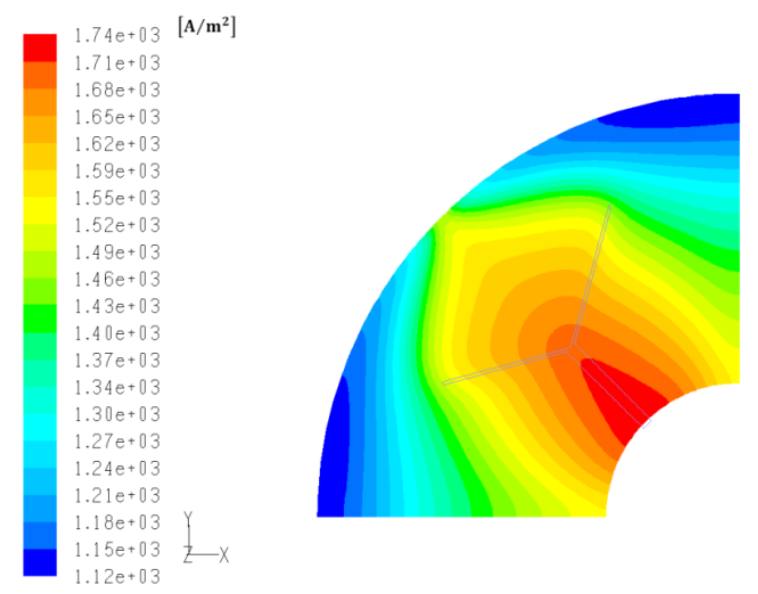

Figure 16. Current density distribution (figure is in color in the on-line version of the paper). 

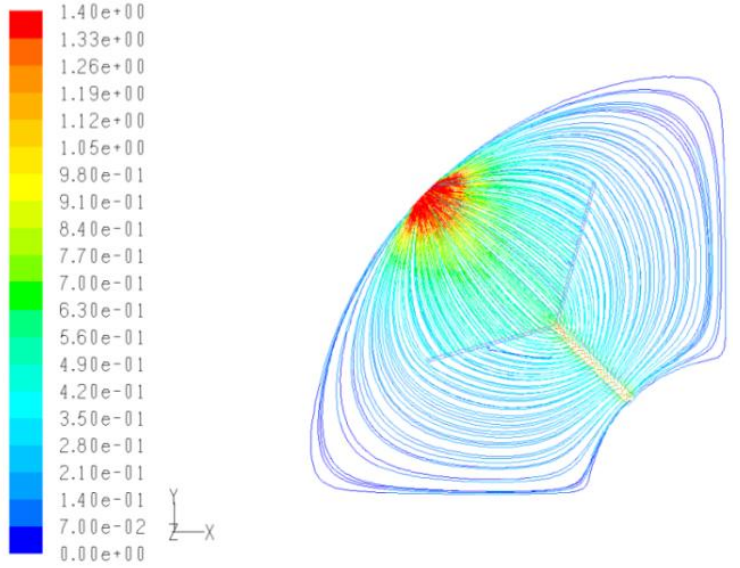

Figure 17. Pathlines colored by velocity magnitude $(\mathrm{m} / \mathrm{s})$ (figure is in color in the on-line version of the paper).

The velocity field is also affected by this configuration; the pathline patterns are illustrated in Figure 17. Fluid departs from the inlet channel, consequently higher velocities are achieved in the middle region on the fuel cell. Moreover, this channel allows one to reduce the size of the stagnation regions inside the cell, which permits to obtain more homogeneous conditions inside the system.

\section{Entropy Generation Analysis}

The distribution inside the fuel cell of the various contributions to the entropy generation are analyzed in this section. Local rate of entropy generation due to viscous effects in the anodic compartment of the cell is depicted in Figure 18. The largest values of $g_{\mu}$ are located where there are strong velocity gradients. In particular, they take place in a confined region close to the anodic compartment outlet. It is clear from Figure 17 that in this region the fluid velocity increases because of the relatively small outlet cross-section, therefore here are located the strongest velocity gradients.

The contribution to entropy generation due to mass transfer is shown in Figure 19. The electrochemical reactions cause strong species mass fraction gradients. Moreover, because of the presence of porous matrix, the species motion in the fuel cell is mainly driven by diffusion, i.e. concentration gradient. These gradients are the sources of entropy generation. The species mass fractions are uniformly distributed in the area spanned by the $\mathrm{Y}$-shaped inlet channel. For this reason, irreversibilities due to mass transfer are negligible in this zone of the fuel cell. In contrast, the outer region of the fuel cell is not reached by the inlet channel. It follows that in this part of the cell chemical species are distributed only by means of diffusion, consequently mass transfer entropy generation is large. This is particularly evident for the ends of secondary inlet channel branches. In the outlet section region convective transport becomes no more negligible, therefore, chemical species are strongly advected. This fact, together with the presence of the outer wall, causes strong species gradients, thus large entropy generation.

The entropy generated by coupling between heat and mass transfer is depicted in Figure 20. This contribution is uniformly distributed in the fuel cell. Largest values are located along the inlet channel profiles because of the contemporary presence of temperature gradients and species concentration gradients. On the other hand, in the proximity of fuel cell supporting structure $g_{c}$ is zero, since adiabatic conditions are considered, therefore temperature gradient is zero.
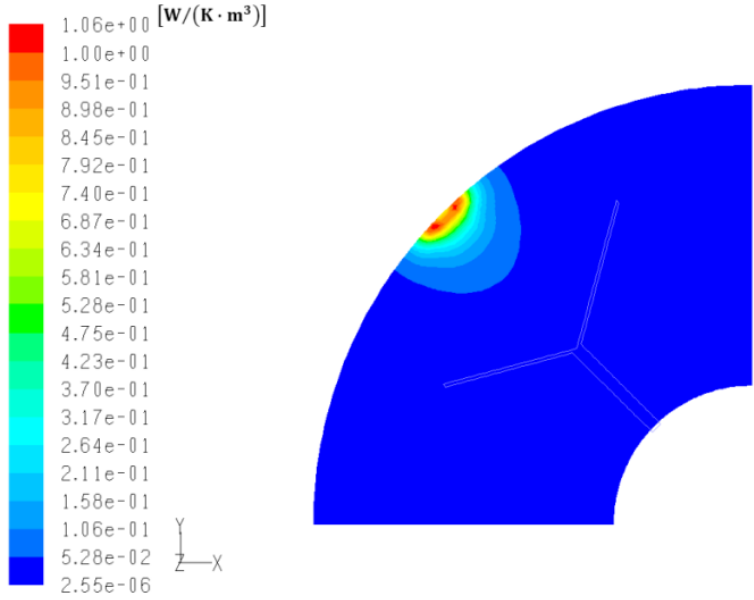

Figure 18. Viscous entropy generation in the anodic channel (figure is in color in the on-line version of the paper).
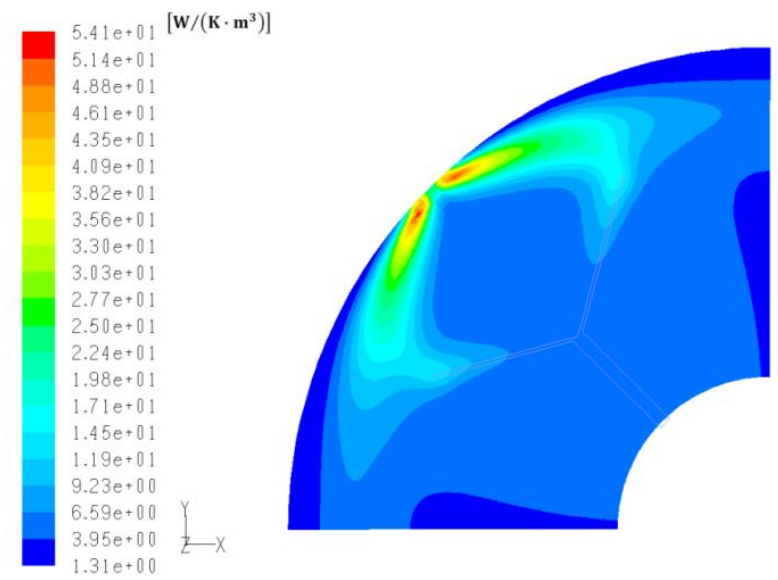

Figure 19. Mass transfer entropy generation (figure is in color in the on-line version of the paper).
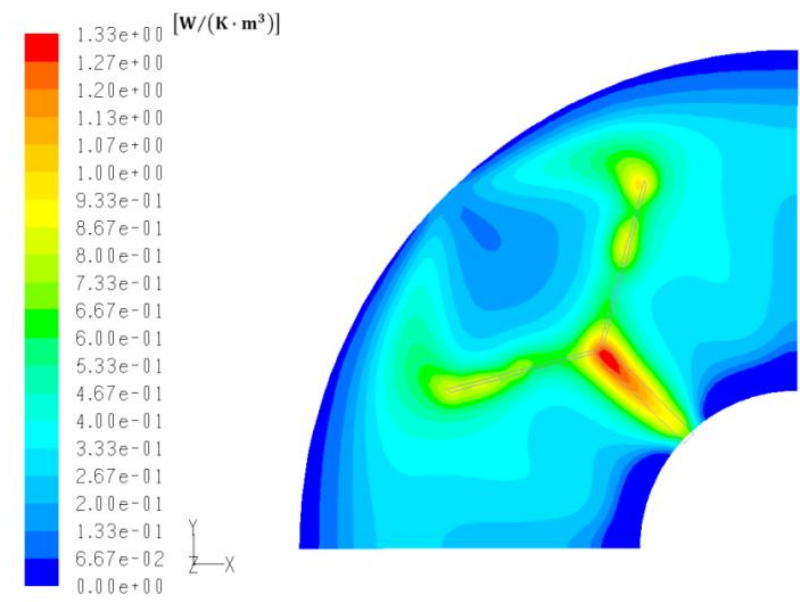

Figure 20. Entropy generation due to coupling between heat and mass transfer (figure is in color in the on-line version of the paper).

The entropy generation rate over the entire domain is investigated during the optimization procedure. Each component of the global entropy generation can be evaluated as follow:

$G_{i}=\int g_{i} d V$

where $g_{i}$ are the contributions to the entropy generation. In Eqn (31), the integral is extended to the whole domain of the fuel cell. In Figure 21, a comparison between each of overall 
entropy generation components is shown. The irreversible losses due to fluid friction are completely negligible since the corresponding specific term is relevant only in a small region of the fuel cell, as shown in Figure 18. The global entropy generation due to coupling between heat and mass transfer is the second most relevant contribution. This can be understood by looking at the distribution represented in Figure 20. This kind of loss is present in the majority of the fuel cells, however since the magnitude of $g_{c}$ is small, it follows that the global term $G_{c}$ is not preponderant. As shown in Figure 21, the main contribution to the total entropy generation is due to mass transfer irreversibility. This is due to diffusion, which is not negligible in the entire domain of the fuel cell, since convective transport is limited by the presence of porous matrix. This causes $g_{m}$ to be relevant in the entire domain and particularly in the outer region of the fuel cell. As a result the global entropy production due to mass transfer is the most important. Additional reductions of this term may be achieved using distribution channels also on the cathodic side, which means that the fuel cell performance can be further increased.

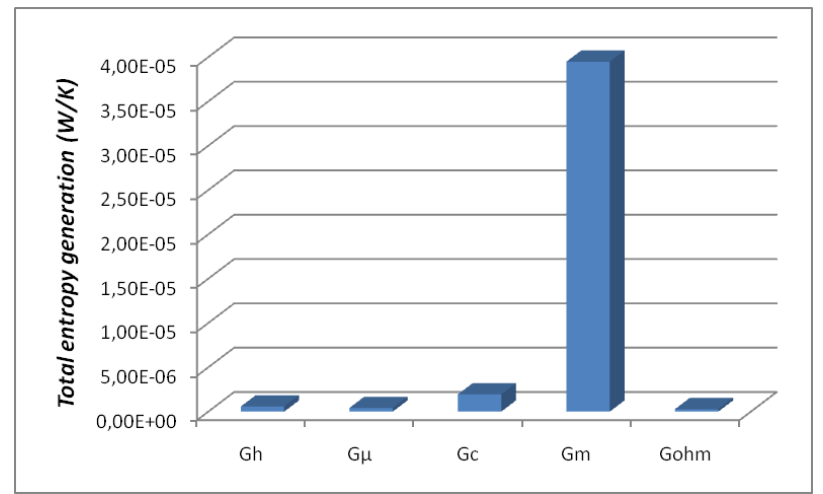

Figure 21. Global entropy generation.

\section{Conclusion}

In this paper entropy generation analysis is used in order to optimize the fluid distribution in a molten carbonate fuel cell. First the architecture of the distribution channels is selected using a network model and then the final design is conducted using a CFD model of the cell run through an optimization algorithm.

Results show that the entropy generation analysis is particularly useful for such an application. With respect to the initial design, the optimized configuration allows an increase in the average current density of about $7 \%$. The analysis is conducted considering distribution channels on the anodic side only. Entropy generation analysis shows that the largest contribution to the irreversibilities is due to mass transfer in the porous media. This means that additional improvements are expected using distribution channels on the cathodic side.

\begin{tabular}{ll}
\multicolumn{2}{l}{ Nomenclature } \\
$\boldsymbol{A}$ & Incidence matrix (-) \\
$D_{i, \text { eff }}$ & Effective binary diffusivity \\
$\mathrm{E}$ & Total energy $\left(\mathrm{J} \mathrm{kg}^{-1}\right)$ \\
$\mathrm{F}$ & Faraday Constant $\left(\mathrm{C} \mathrm{mol}^{-1}\right)$ \\
$\mathrm{H}$ & Specific enthalpy $\left(\mathrm{J} \mathrm{kg}^{-1}\right)$ \\
$G_{i}$ & Global entropy generation $\left(\mathrm{W} \mathrm{K}^{-1}\right)$ \\
$\mathrm{I}$ & Current density $\left(\mathrm{A} / \mathrm{m}^{2}\right)$ \\
$\vec{J}_{i}$ & Diffusive flux of species $i\left(\mathrm{~kg} \mathrm{~m}^{-2} \mathrm{~s}^{-1}\right)$
\end{tabular}

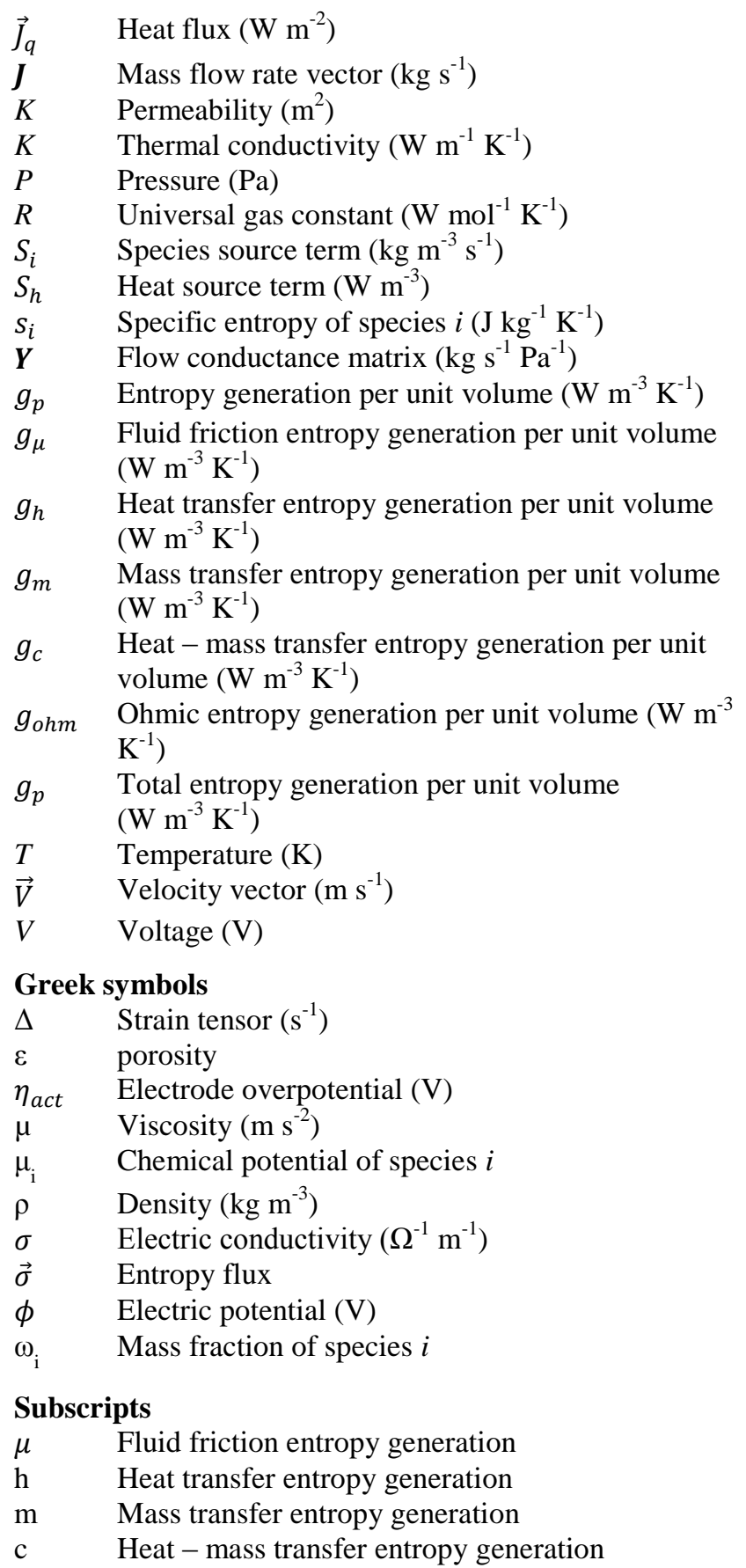

\section{References:}

Amelio C., Diaz G., Ferrari E., Ghisolfi E., L. Mannarino L., Spadaro C., Poskovic E. \& Baccaro S. (2009). A New Approach to MCFC Ceramic Matrixes Manufacturing and Laboratory Small scale Stack Testing, Development of Innovative SOFC Cell Porous Components. European Fuel Cell Conference. Paper EFC09-17166.

Bejan A. Entropy generation minimization. Boca Raton: CRC; 1996.

Bejan A., Lorente S. (2004). The constructal law and the thermodynamics of flow systems with configuration, International Journal of Heat and Mass Transfer 47: 3203-3214.

Bittanti S, Canavese S, De Marco A, Errigo A, Prandoni V. (2006). Molten Carbonate Fuel Cell electrochemistry modelling. Journal of Power Sources 160.846 - 851. 
Calì M., Borchiellini R., (2002). District Heating Network Calculation and Optimization. Encyclopedia of Life Support Systems, UNESCO (paper 3.19.3.8).

Chandrashekar M., Wong F. C. (1982). Thermodynamic System Analysis - A graph-theoretic Approach. Energy, Vol.7 No.6, pp.539-566.

Cigolotti V, Massi E, Moreno A, Pollettini A, Reale F. Biofuels as opportunity for MCFC niche market application. International Journal of Hydrogen Energy 33. $2999-3003$.

Copiello D., Fabbri G. (2009). Multi-objective genetic optimization of the heat transfer from longitudinal wavy fins, International Journal of Heat and Mass Transfer 52: $1167-1176$

Fontes E, Fontes M, Simonsson D. (1995) A heterogeneous model for the MCFC cathode. Electrochimical Acta 40. 1641-1647.

Hwang JJ, Chen CK, Lai DY. (2005). Computational analysis of species transport and electrochemical characteristics of a MOLB-type SOFC, Journal of Power Sources. 140: 235-42.

Iandoli L, Sciubba E (2005). 3-D Numerical Calculation of the Local Entropy Generation Rates in a Radial Compressor Stage. Int. J. of Thermodynamics, 8: 83-94

Ibáñez G., Cuevas S. (2010). Entropy generation minimization of a MHD (magnetohydrodynamic) flow in a microchannel. Energy 35. 4149-4155.

Kalyanmoy Deb (2002). A Fast and Elitist Multiobjective Genetic Algorithm, IEEE TRANSACTIONS ON EVOLUTIONARY COMPUTATION, Vol. 6, No. 2, pp. 182-197.

Koh J.H., Seo H.K, Yoo Y.S \& Lim H.C. (2002). Consideration of numerical simulation parameters and heat transfer models for a molten carbonate fuel cell stack, Chemical Engineering Journal, Vol. 87, pp. 367379.

Krishna R., J. A. Wesselingh J. A. (1996). The MaxwellStefan approach to mass transfer, Chemical Engineering Science, Vol. 52, No. 6, pp. 861-911.

NETL (2004). Fuel Cell Handbook, seventh ed. US Department of Energy, National Energy Technology Laboratory, Morgantown.

Nield D.A., Bejan A. (1999). Convection in porous media. New York: Springer.

Ma Z, Jeter SM, Abdel-Khalik SI. (2003). Modeling the tran sport processes within multichannel molten carbonate fuel cells. International Journal of Hydrogen Energy 28. 85-97.

Massano C., Sciacovelli A. \& Verda V. (2009). Detailed Model of Molten Carbonate Fuel Cell Stacks. ECOS 2009. August 31 - September 3, 2009, Foz do Iguaçu, Paraná, Brazil.

Marra D. (2008). Gas distribution inside an MCFC. International Journal of Hydrogen Energy 33. 3173 3177.

Park HK, Lee Y-R, Kim M-H, Chung G-Y, Nam S-W, Hing S-A. (2002). Studies of the effects of the reformer in an internal reforming molten carbonate fuel cell by mathematical modeling. Journal of Power Sources 104. $140-7$.

Patankar SV. (1980). Numerical heat transfer and fluid flow. Hemisphere Publishing Corporation.

Raghavan V., Gogos G., Babub V., Sundararajan T. (2007) Entropy generation during the quasi-steady burning of spherical fuel particles. International Journal of Thermal Sciences 46. 589-604.

Revellin R., Lips S., Khandekar S, Jocelyn Bonjour J. (2009) Local entropy generation for saturated two-phase flow. Energy 34. 1113-1121.

San JY. (2010). Second-law performance of heat exchangers for waste heat recovery. Energy 35. 1936 - 1945.

Sciacovelli A., Verda V. (2009) Entropy generation analysis in a monolithic-type solid oxide fuel cell (SOFC), Energy 34. 850-865.

Verda V., Nicolin F. (2010). Thermodynamic and economic optimization of a MCFC-based hybrid system for the combined production of electricity and hydrogen. International Journal of Hydrogen Energy 35. 794 -806.

V. Verda, A. Sciacovelli (2011). Design improvement of circular molten carbonate fuel cell stack through CFD Analysis, Applied Thermal Engineering, doi:10.1016/j.applthermaleng.2011.04.046

Xing XQ., Lum KW., Poh HJ., Wu YL. (2009). Geometry optimization for proton-exchange membrane fuel cells with sequential quadratic programming method, Journal of Power Sources 186, 10-21.

Zimparov V.D., da Silva A.K. \& Bejan A. (2006). Thermodynamic optimization of tree-shaped flow geometries, International Journal of Heat and Mass Transfer 49: 1619-1630. 